\title{
Integrin-linked kinase pathway in heterogeneous pulmonary sarcomatoid carcinoma
}

\author{
SHIGEKI SHIMIZU $^{1 *}$, KAZUKO SAKAI $^{2 *}$, TAKAAKI CHIKUGO ${ }^{1}$, TAKAO SATOU $^{1}$, \\ NAOKI SHIRAISHI $^{1}$, TETSUYA MITSUDOMI ${ }^{3}$ and KAZUTO NISHIO $^{2}$ \\ ${ }^{1}$ Department of Diagnostic Pathology, Kindai University Hospital; ${ }^{2}$ Department of Genome Biology, \\ Kindai University Faculty of Medicine; ${ }^{3}$ Department of Thoracic Surgery, \\ Kindai University Faculty of Medicine, Osaka-Sayama, Osaka 589-8511, Japan
}

Received July 25, 2020; Accepted December 14, 2020

DOI: $10.3892 / \mathrm{ol} .2021 .12582$

\begin{abstract}
Pulmonary sarcomatoid carcinoma (PSC) is classified as poorly differentiated, and non-small cell lung carcinomas that contained a component of sarcoma or sarcoma-like differentiation are rare. The underlying carcinogenetic mechanism governing PSC remains unclear. The current study investigated the underlying carcinogenetic mechanism of PSC based on the hypothesis that it involves the epithelial-mesenchymal transition (EMT) process. Mutation analysis of PSCs, including carcinosarcoma, pleomorphic carcinoma and epithelial carcinoma specimens, was performed using targeted deep sequencing, whole transcriptome analysis and digital spatial profiling (DSP). PSCs exhibit a distinct mutation profile, with TP53, SYNE1 and APC mutations. Therefore, clustering of the gene expression profiles allowed the PSCs to be distinguished from the epithelial carcinomas. Increased gene expression of fibronectin in PSC was an important contributor to differential profiles. Pathway analysis revealed enhanced activity of the integrin-linked kinase (ILK) signaling pathway in the PSCs. DSP analysis using 56 antibodies of marker proteins confirmed significantly higher expression of fibronectin in PSCs. Intratumor heterogeneity of fibronectin expression was observed in sarcoma components. In conclusion, epithelial-mesenchymal transition process mediated by ILK signaling may be associated with carcinogenetic mechanisms of PSC. Overexpression of fibronectin mediated by ILK signaling appears to serve a role in the EMT involved in the PSC transformation process.
\end{abstract}

Correspondence to: Dr Kazuto Nishio, Department of Genome Biology, Kindai University Faculty of Medicine, 377-2 Ohno-Higashi, Osaka-Sayama, Osaka 589-8511, Japan

E-mail: knishio@med.kindai.ac.jp

${ }^{*}$ Contributed equally

Key words: carcinosarcoma, digital spatial profiling, deep sequencing, pathway analysis, epithelial-mesenchymal transition

\section{Introduction}

Pulmonary sarcomatoid carcinomas (PSCs) are a rare subset of lung tumors, with an estimated incidence of $<1 \%$ of all primary lung neoplasms (1-3). PSCs are currently defined as poorly differentiated non-small cell carcinomas containing a component with sarcoma or sarcoma-like (spindle and/or giant cell) features (4). Five major histological variants have been described, namely pleomorphic carcinoma, spindle cell carcinoma, giant cell carcinoma, carcinosarcoma, and pulmonary blastoma. Carcinosarcomas contain ectopic components (bone, chondrosarcoma, and rhabdomyosarcoma) as the non-epithelial components. Patients with PSCs generally show an aggressive clinical course. PSCs are considered to be in an 'in transition' status, at the crossroads of diverse pathways of clonal evolution. The sarcomatous or sarcomatoid components in these tumors may be derived from carcinoma cells through the activation of an epithelial-mesenchymal transition (EMT) process that leads to sarcomatous transformation or metaplasia of the carcinoma cells.

The epithelial nature of sarcomatoid carcinomas is highlighted by the expression of pan-epithelial markers, such as pan-cytokeratin AE1/AE3, OSCAR and keratin, both in the differentiated elements and the spindle or giant cells of these tumors $(5,6)$. In the rare cases in which the sarcomatoid components of the tumors tested negative for pan-epithelial markers, positive results are often obtained for other markers associated with epithelial tumors (e.g., CK7, TTF-1 and p40). The majority of sarcomatoid carcinomas also express markers of more specific differentiation. Expressions of markers related to adenocarcinomatous differentiation, including CK7, TTF-1 and napsin A, have been demonstrated in up to 78,61 and $39 \%$ of PSCs, respectively $(5,6)$. A subset of sarcomatoid carcinomas is characterized by the expressions of p40, CK5/6, Sox 2 and/or desmocollin-3 $(4,6,7)$ but reactivity for these markers is generally lower than that of tumors with an adenocarcinoma phenotype $(5,6)$. A wider panel of immune-markers is therefore required. The NanoString digital spatial profiling technology allows identification using a specially defined collection of oligonucleotide tags that are cleaved from specific validated antibodies (8). The spots of interest may be user-defined (drawn on an image) or 
molecularly defined using fluorescence images of the same slide prior to collection. In the present study, formalin-fixed paraffin-embedded (FFPE) tissue sections of PSC were incubated with cocktails of 56 unique oligonucleotide-conjugated antibodies and analyzed.

The precise molecular characteristics of sarcomatoid carcinomas are largely unexplored. In this context, TP53 and KRAS mutations are reported as being among the most common genomic alterations in sarcomatoid carcinomas (up to 74 and $34 \%$ of cases, respectively), the latter likely triggered by tobacco use $(2,5,9)$. In addition, targetable oncogenic driver mutations, such as EGFR, BRAF, HER2, RET, ALK, AKT1, $J A K 3, N R A S$ or PIK3CA, have also been identified in a small but consistent subset of PSCs $(5,9)$. Recent progress in deep sequencing technology has enabled concurrent analyses of gene mutation profiling and transcriptome. Actionable mutations are somewhat less frequent in sarcomatoid carcinomas as compared to non-small cell lung cancer (NSCLC) (6). In this study, we attempted to profile the molecular statuses of the same PSC-FFPE samples at the genome, transcript and protein levels.

\section{Materials and methods}

Clinical specimens. Tumor specimens of PSCs and NSCLC were obtained from patients seen at the Kindai University Faculty of Medicine, with the approval of the Institutional Review Board (30-034). Written informed consent was obtained from each patient.

Nucleic acid extraction. DNA and RNA were purified with the use of an Allprep DNA/RNA FFPE kit (Qiagen, Inc.) according to the manufacturer's instructions. The quality and quantity of the DNA/RNA were verified using the NanoDrop 2000 device (Thermo Fisher Scientific, Inc.), PicoGreen dsDNA assay kit (Life Technologies; Thermo Fisher Scientific, Inc.) and the RiboGreen RNA assay kit (Life Technologies; Thermo Fisher Scientific, Inc.). The extracted DNA/RNA was stored at $-80^{\circ} \mathrm{C}$ until the analysis.

Targeted DNA sequencing. For DNA sequencing, $40 \mathrm{ng}$ of DNA were subjected to multiplex PCR amplification with the use of an Ion AmpliSeq Library Kit 2.0 and Ion AmpliSeq ${ }^{\mathrm{TM}}$ Comprehensive Cancer Panel (CCP, Thermo Fisher Scientific, Inc.), covering all exon in 409 genes. After multiplex PCR, Ion Xpress Barcode Adapters (Thermo Fisher Scientific, Inc.) were ligated to the PCR products, which were then purified with the use of Agencourt AMPure XP beads (Beckman Coulter, Inc.). The purified libraries were pooled and then sequenced with the use of an Ion Torrent S5 instrument and Ion 550 Chip Kit (all from Thermo Fisher Scientific, Inc.). DNA sequencing data were accessed through the Torrent Suite v.5.10 program (Thermo Fisher Scientific, Inc.). Reads were aligned against the hg19 human reference genome, and variants were called with the use of Variant Call Format ver. 5.10. Raw variant calls were filtered with quality score of $<100$, depth of coverage of $<19$, and were manually checked using the integrative genomics viewer (IGV; Broad Institute, Cambridge, MA, USA). Germline mutations were excluded with the use of the Genome Aggregation Database (gnomAD).
Whole transcriptome sequencing. For library preparation, a barcoded cDNA library is first generated with SuperScript VILO cDNA Synthesis kit (Thermo Fisher Scientific, Inc.) from $10 \mathrm{ng}$ of total RNA. Then cDNA is prepared using the AmpliSeq Transcriptome Human Gene Expression kit (Thermo Fisher Scientific, Inc.) according to the manufacturer's instructions. Pooled libraries were subjected to the Ion Chef System (Thermo Fisher Scientific, Inc.) for template preparation. Libraries were then loaded onto an Ion 550 chip and sequenced with the Ion S5 sequencing system. The Ion Torrent Suite v5.10 software (Thermo Fisher Scientific, Inc.) was used for base calling, alignment to the human reference genome (hg19) and quality control. Raw reads were then analyzed automatically using the AmpliSeqRNA plugin to generate gene-level expression values for all 20802 RefSeq human genes. Genes with a fold change greater than 4 (the absolute value of $\log 2$ fold change greater than 2) and an adjust P-value $<0.05$ were considered as differentially expressed and were investigated by Ingenuity ${ }^{\circledR}$ Pathway Analysis (IPA) (Qiagen, Inc.). The datasets used and analyzed during the current study are available from the corresponding author on reasonable request.

Digital spatial profiling. The NanoString digital spatial profiling technology allows identification using a specially defined collection of oligonucleotides tags that are cleaved from specific validated antibodies $(8,10)$. The regions of interest may be user-defined (drawn on an image) or molecularly defined using a fluorescence 152 image of the same slide prior to collection. The FFPE tissue sections were incubated with cocktails of 56 unique oligonucleotide-conjugated antibodies (Table SI). The selected compartments were chosen for high-resolution multiplex profiling, and oligos from the selected region were released upon exposure to UV light. Photocleaved oligos were then collected via microcapillary tube inspiration using an early version of the DSP platform (NanoString) robotic system and transferred into a microwell plate with a partial resolution of approximately $10 \mu \mathrm{m}$. Photocleaved oligos from the spatially-resolved compartments in the microplate were then hybridized to 4-color, 6-spot optical barcodes in the nCounter ${ }^{\circledR}$ platform, enabling up to 800 distinctly labels counts per compartment of the protein targets representing the antibodies to which the tags were originally conjugated. Digital counts from barcodes corresponding to the protein probes were first normalized with internal spike-in controls (ERCCs) to account for system variations, and then normalized to immunoglobulin $\mathrm{G}$ (IgG) controls to correct for noise.

Statistical analysis. Statistical analysis was performed using GraphPad Prism software (ver. 8, GraphPad Software Inc.). The data represent the means \pm standard deviation (SD). The difference between groups was calculated using Mann-Whitney U test. Correlation between cell component and 56 protein expression by DSP was calculated using $\chi^{2}$ test. A P-value of $<0.05$ was considered statistically significant.

\section{Results}

Somatic mutation analysis. DNA extracted from FFPE tissue samples obtained from patients histopathologically diagnosed 


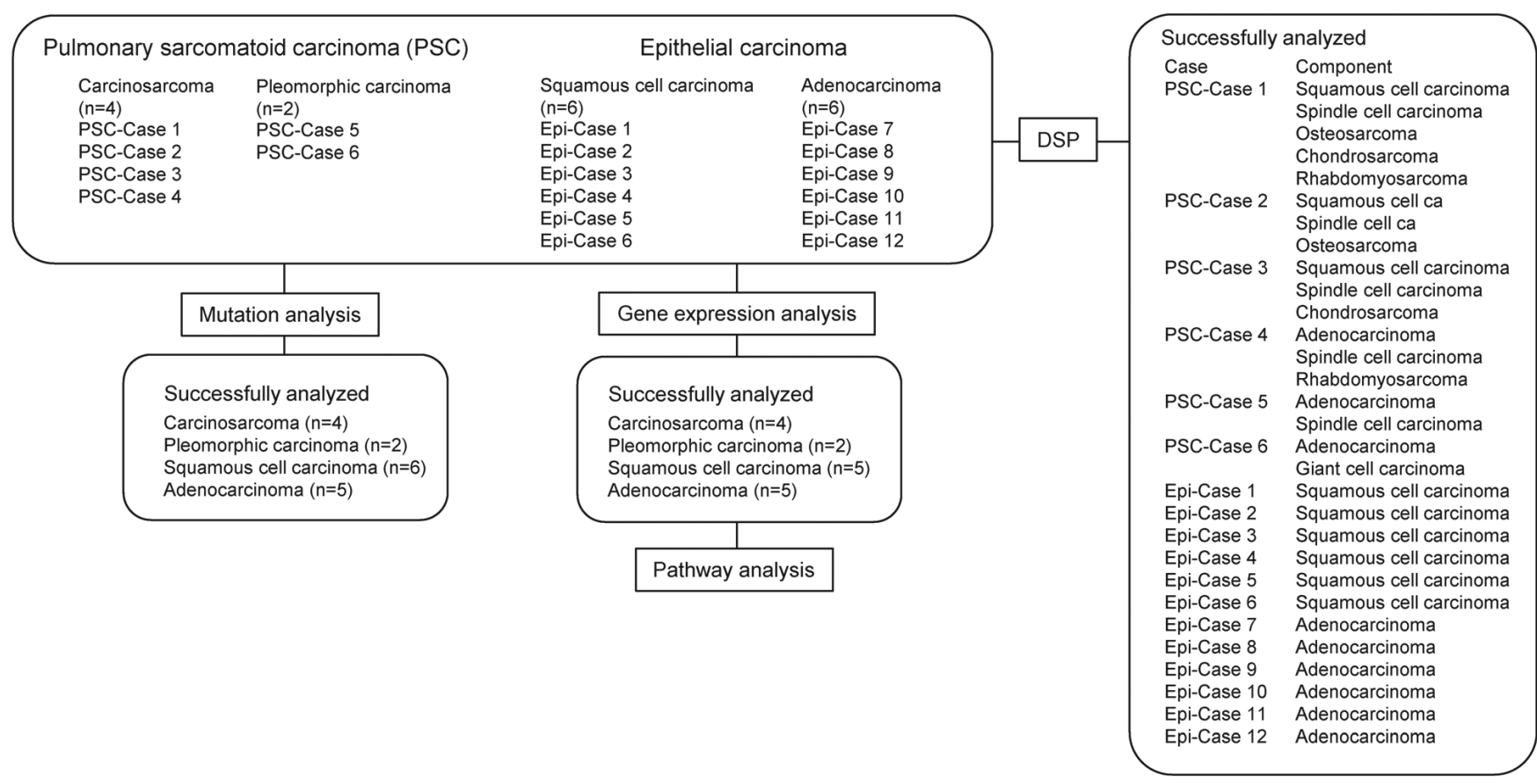

Figure 1. Analytical flow of PSC and epithelial carcinomas (Epi). CCP, comprehensive cancer panel; DSP, digital spatial profiling; PSC, Pulmonary sarcomatoid carcinoma; Epi, epithelial carcinomas.

as having PSC at Kindai University Hospital from 2016 to 2018 were analyzed. Samples from 6 lung adenocarcinoma and 6 squamous cell lung cancer patients were processed as the epithelial carcinoma samples (Fig. 1). No analysis was performed in one of the lung adenocarcinoma case, because of the low quality of DNA isolated. PSC-case 1 was a 63-year-old male patient, and the tumor tissue contained squamous cell carcinoma, spindle cell carcinoma, osteosarcoma, chondrosarcoma, and rhabdomyosarcoma components (Fig. S1). PSC-case 2 was a 71-year-old female patient and her tumor tissue contained squamous cell carcinoma, spindle cell carcinoma and osteosarcoma components. PSC-case 3 was a 79-year-old male patient and his tumor tissue contained squamous cell carcinoma, spindle cell carcinoma, chondrosarcoma, and giant cell carcinoma components. PSC-case 4 was an 86-year-old male patient, and his tumor tissue contained typical adenocarcinoma, high-grade fetal carcinoma-like adenocarcinoma, spindle cell carcinoma, and rhabdomyosarcoma components. All patients had a history of smoking. Targeted deep sequencing with CCP for 409 cancer-related genes revealed 7, 7, 6, and 5 pathogenic mutations classified by Functional Analysis through Hidden Markov Models (FATHMM) in PSC-cases 1-4, respectively (Fig. S2A). Mutations of TP53 (3/4), SYNE1 (2/4), and APC $(2 / 4)$ were detected in the carcinosarcoma. These mutations have been previously recognized in carcinosarcoma (5), but are not found in pleomorphic carcinoma. On the other hand, TP53 mutation was also detected in 4/11 epithelial carcinoma (adenocarcinoma and squamous cell carcinoma) cases. CCP panel detected likely pathogenic mutations such as NTRK3 and PIK3CA in PSC-case 1, AXL and KDR in PSC-case 2, PTEN in PSC-case 3 , and $B R A F$ in PSC-case 4 . The non-synonymous tumor mutation burden (TMB), which is a predictive marker for response to immunocheckpoint inhibitor therapy (11-13), was calculated from CCP panel (Fig. S2B). The average TMB of the PSCs was 15.2 mutations/Mb, which was nearly as high as that in epithelial carcinomas (adenocarcinoma + squamous cell carcinoma, 12.8 mutations/Mb), though the difference was not statistically significant.

Gene expression profile. Whole-transcriptome analysis was performed for the 6 PSC (PSC-Cases 1-6), 6 adenocarcinoma and 6 squamous cell carcinoma (Epi-Cases 1-6) specimens (Fig. 1). No analysis was performed in two of the epithelial carcinomas (1 adenocarcinoma and 1 squamous cell carcinoma) cases, because of the low quality of RNA isolated. Clustering analysis allowed the specimens to be clearly classified into epithelial carcinoma and PSC based on the gene expression profile (Fig. 2A). Pathway analysis (IPA) revealed significant enrichment of pathways of PSCs (Fig. 2B). The top 20 pathways included the integrin-linked kinase (ILK) signaling pathway, which is known to be related to $\beta$-catenin-mediated EMT (14) through AKT and Gsk3 $\beta$ (15). ILK signaling activates the key transcription factor SLUG (SNAI2), which upregulates the expression of fibronectin as well as vimentin involved in the EMT process (16). Fibronectin acts on cell mobility and cell adhesion. The gene expression levels of fibronectin as well as vimentin and SNAI2 were higher in the PSCs than in the epithelial carcinomas (Fig. 3). The fibronectin gene expression levels were also higher in the PSCs than in the squamous cell carcinomas.

Intratumor heterogeneity of fibronectin expression in pulmonary sarcomatous carcinomas. Standard immunohistochemistry (IHC) was performed for the PSC specimens (Table I). With regards to E-cadherin expression, carcinoma components show positive staining, whereas sarcomatous components, including spindle cell carcinoma, osteosarcoma, chondrosarcoma, rhabdomyosarcoma, in each sample are 

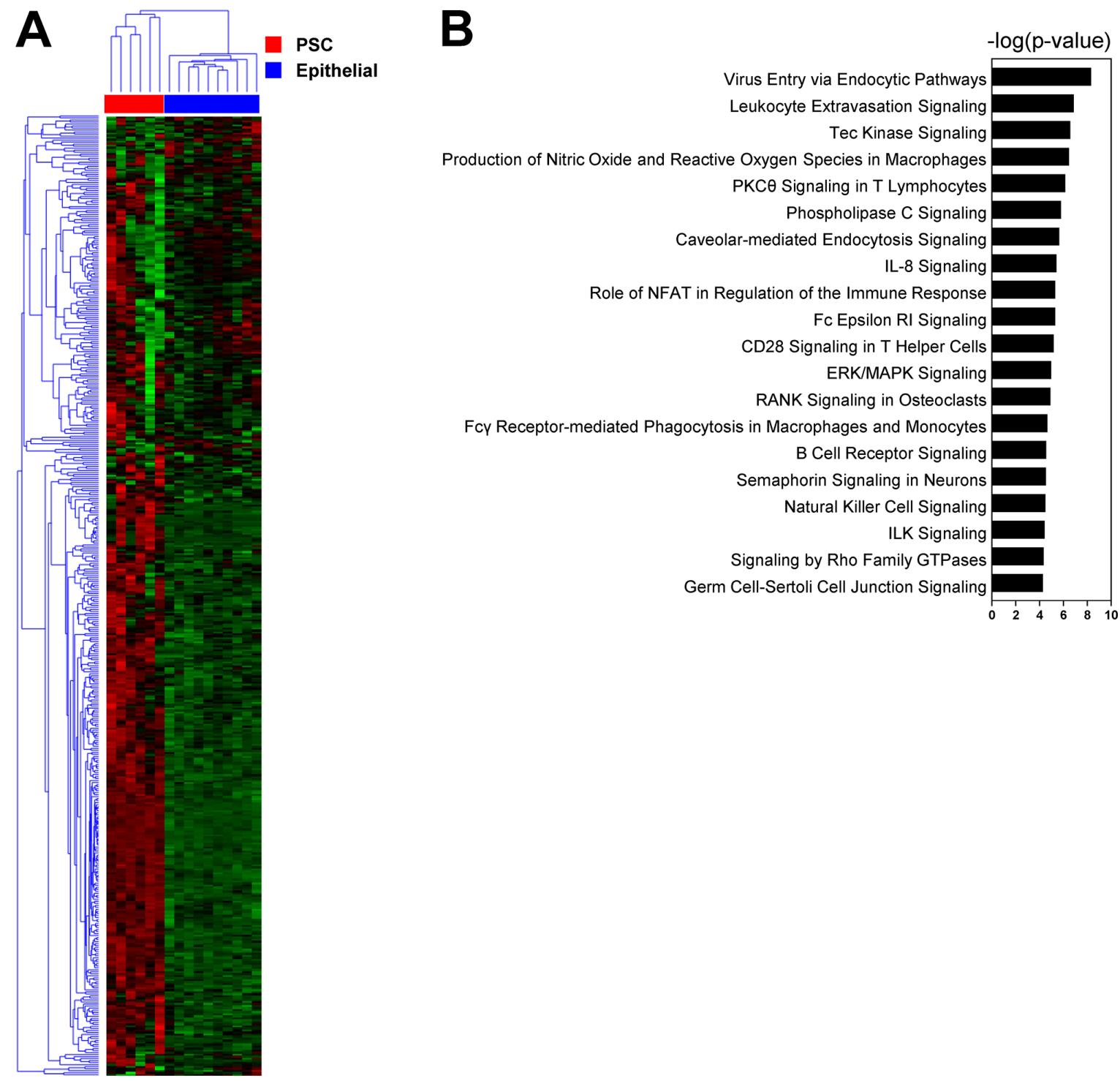

Figure 2. Whole-transcriptome analysis of PSCs. Clustering, Z-scoring of each gene, and pathway analysis was performed by Ingenuity pathway analysis (IPA; Qiagen Redwood City, http://www.qiagen.com/ingenuity). (A) Clustering analysis allowed the carcinomas (Epithelial) to be clearly distinguished from the PSCs. PSCs (4 sarcoma and 2 pleomorphic carcinoma) were compared with epithelial carcinomas (5 adenocarcinoma and 5 squamous cell carcinoma). (B) Pathway analysis showed the 20 top pathways including the ILK signaling pathway. PSC, Pulmonary sarcomatoid carcinoma; ILK, integrin-linked kinase.
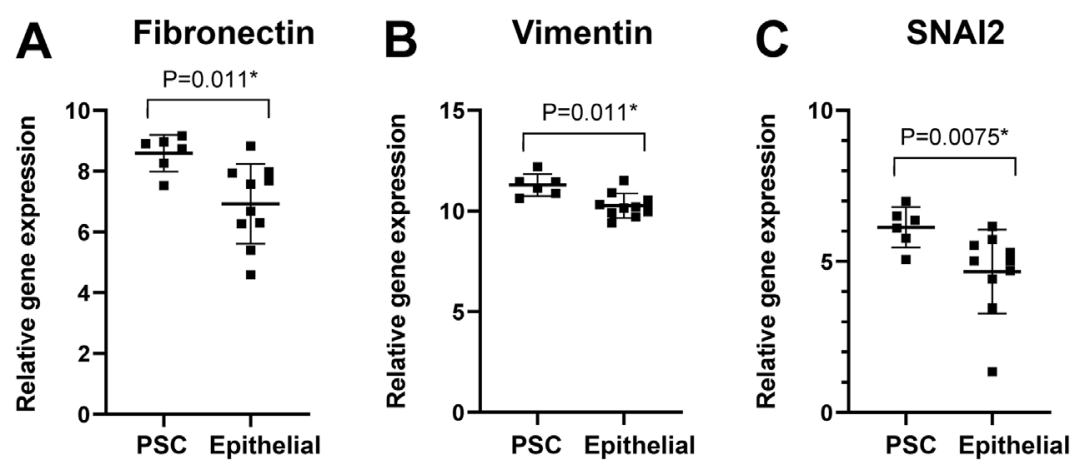

Figure 3. Gene expression levels of (A) fibronectin, (B) vimentin and (C) SNAI2 in the epithelial carcinomas (5 adenocarcinoma and 5 squamous cell carcinoma) and pulmonary sarcomatoid carcinomas (PSCs) (4 sarcomas and 2 pleomorphic carcinomas). Gene expression levels were determined by whole-transcriptome assay. The data represent the mean $\pm \mathrm{SD}$. ${ }^{*} \mathrm{P}<0.05$. PSC, Pulmonary sarcomatoid carcinoma.

negative. E-cadherin expression patterns were consistent across all cases. To further investigate the biological features and intratumor heterogeneity of PSCs, digital IHC analysis by DSP was performed for 60 selected regions of interest (Fig. S3, 
Table I. Detailed clinical features in 4 patients with carcinosarcoma.

A, PSC Case 1

Component

IHC

Squamous cell carcinoma AE1/AE3(+), E-cadherin(+), TTF-1(+), p40(+), SMA(-), Desmin(-), Myogenin(-)

Spindle cell carcinoma

AE1/AE3(focal +), E-cadherin(-), TTF-1(+), p40(+), SMA(focal +), Desmin(-), Myogenin(-)

Osteosarcoma

AE1/AE3(-), E-cadherin(-), TTF-1(-), p40(-), SMA(-), Desmin(-), Myogenin(-)

Chondrosarcoma

AE1/AE3(-), E-cadherin(-), TTF-1(-), p40(-), SMA(-), Desmin(-), Myogenin(-)

Rhabdomyosarcoma

AE1/AE3(-), E-cadherin(-), TTF-1(-), p40(-), SMA(-), Desmin(+), Myogenin(+)

\section{B, PSC Case 2}

Component

$\mathrm{IHC}$

Squamous cell carcinoma AE1/AE3(+), E-cadherin(+), TTF-1(focal +), p40(+), CK5/6(+), SMA(-), CD56(-), S100(-)

Spindle cell carcinoma

AE1/AE3(-), E-cadherin(-), TTF-1(-), p40(-), CK5/6(-), SMA(+), CD56(+), S100(focal +)

Osteosarcoma

AE1/AE3(-), E-cadherin(-), TTF-1(-), p40(-), CK5/6(-), SMA(-), CD56(+), S100(-)

\section{C, PSC Case 3}

Component

IHC

Squamous cell carcinoma AE1/AE3(+), E-cadherin(+), TTF-1(-), p40(+), SMA(-), CD56(-), Desmin(-), S100(-)

Spindle cell carcinoma AE1/AE3(-), E-cadherin(-), TTF-1(-), p40(+), SMA(-), CD56(-), Desmin(-), S100(-)

Chondrosarcoma

AE1/AE3(-), E-cadherin(-), TTF-1(-), p40(-), SMA(-), CD56(-), Desmin(-), S100(+)

D, PSC Case 4

Component

Adenocarcinoma

Spindle cell carcinoma

Rhabdomyosarcoma
IHC

AE1/AE3(+), E-cadherin(+), TTF-1(+), p40(focal +), SMA(-), CD56(focal +), AFP(-), SALL4(-), GPC3(-)

AE1/AE3(-), E-cadherin(-), TTF-1(focal +), p40(-), SMA(-), CD56(+), AFP(-), SALL4(-), GPC3(-)

AE1/AE3(-), E-cadherin(-), TTF-1(-), p40(-), SMA(-), CD56(-), Desmin(+), Myogenin(+)

TTF-1, thyroid transcription factor 1; SMA, smooth muscle actin; AFP, $\alpha$-fetoprotein; GPC3, glypican-3; IHC, immunohistochemistry; $\mathrm{CD}$, cluster of differentiation.

Table SII) using 56 antibodies. Quantitative expression levels of 56 proteins in sarcomatoid (29 spots) and epithelial (31 spots) components were compared between epithelial and sarcomatoid comportment (Fig. 4A). Higher expression levels of fibronectin, CD163, and CD56 were observed in the sarcomatoid components significantly $(\mathrm{P}<0.0001$, Chi-squared test $)$ (Fig. 4B). On the other hand, EPCAM, and pan-cytokeratin expressed highly in the epithelial components. Taken together, the fibronectin expression levels were significantly higher in the sarcomatoid components than in the epithelial components of the PSCs.

Differential intratumor expression of fibronectin was observed in the different sarcoma components (spindle cell sarcoma, osteosarcoma, chondrosarcoma, and rhabdomyosarcoma components) in PSC cases 1-4 (Fig. 5); the highest expression of fibronectin was observed in the osteosarcoma components, while modest expression of fibronectin was observed in the spindle cell sarcoma, rhabdomyosarcoma, and chondrosarcoma components. No fibronectin expression was observed in the epithelial components in three of the four cases (Fig. 5). Differences in the intratumor expression of fibronectin seemed to be related to the degree of EMT in the tumors.

\section{Discussion}

PSCs are rare form of NSCLC cancers that are characterized by their aggressive nature and difficulty to treat. In this study, we have performed DNA mutation and whole transcriptome analyses in a small cohort of PSC cases and compared the patients' genomic and transcriptomic profiles to protein expression profiles from digital multiplexed immunohistochemical tissue sections. Molecular profiling of PSCs has been reported by a few previous studies. Terra et al reported that PSCs, 


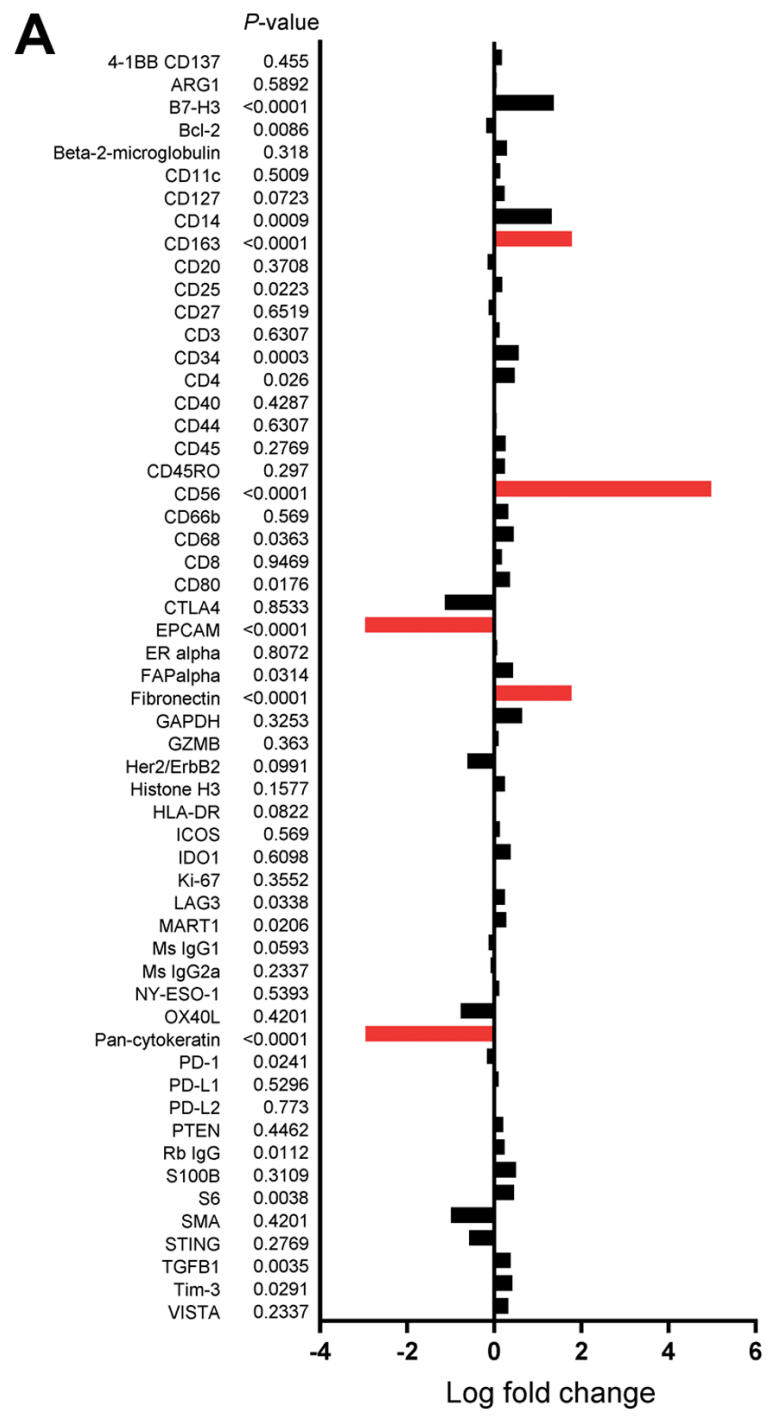

B

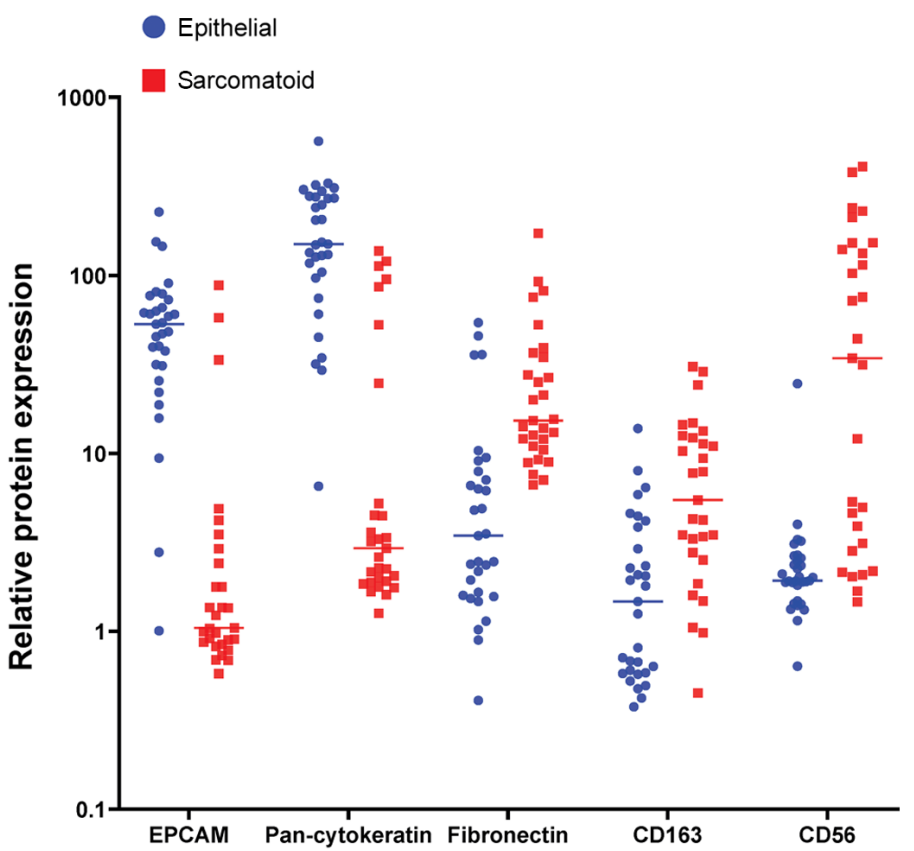

Figure 4. Protein expression levels in epithelial and sarcomatoid components in PSCs and epithelial carcinomas based on protein DSP. (A) Quantitative expression (ratio of signal/noise) of 56 proteins in epithelial (31 spots) and sarcomatoid ( 29 spots) components were analyzed by protein DSP. The ratio of signal/noise was normalized by IgG internal standards. Fold changes of 56 protein expression were shown as $\log 2$ value. P-values were determined by the $\chi^{2}$ test. (B) Five proteins with absolute values of $\log 2$ fold change $>1.5$ and a P-value $\leq .0 .05$ were selected. Dot plot shows the distribution of protein expression for epithelial (blue) and sarcomatoid (red) components. DSP, digital spatial profiling.

consisting of pleomorphic carcinoma, spindle cell carcinoma, carcinosarcoma, and giant cell carcinoma, exhibit mutations of TP53 mutation (58\%), JAK3 (3\%), BRAF (3\%), NRAS (3\%), and PIK3CA (3\%) (5). According to the results of our NGS-based mutation analysis, mutations of TP53 were detected at a high frequency in the PSCs. TP53 mutations are also detected in epithelial carcinomas. No difference in the frequency of TP53 mutations were observed between the PSCs and epithelial carcinoma specimens. Therefore, it is unlikely that they contribute to PSC transformation. Liang et al reported that a high frequency of $R B 1$ mutations (25\%) were detected as well as that of TP53 (69\%) (17). In our cases, no hot spot mutation of $R B 1$ was detected, although data for the full sequence of $R B 1$ was unavailable. NGS analysis also showed a high TMB of PSCs. The TMB was as high in the PSCs as in the NSCLCs. NGS analysis also showed a high TMB of PSCs. The TMB was as high in the PSCs as in the NSCLCs. TMB as well as PD-L1 can be used to predict efficacy of immune checkpoint inhibitors and has become a useful biomarker to identify cancer patients that may likely benefit from immunotherapy. Liang et al reported that $40.6 \%$ (13/32) of Chinese patients with PSC had high TMB (17). This result thus suggests that PSCs have a strong potential to present neoantigens, regardless of the PD-L1 expression status. On the other hand, retrospective studies reported a high incidence of PD-L1 expression in patients with PSC. Velcheti et al reported that $69.2 \%(9 / 13)$ of patients were positive for PD-L1 (18). Taking these findings together, immunotherapy with immune-checkpoint inhibitors could potentially be effective for PSCs, although studies are required prove this assumption.

Pathway analysis (IPA) provided the signaling pathway to identify molecular targets and biomarkers for PSCs. The top 20 significant pathways included the ILK signaling pathway, which is known to be related to beta-catenin-mediated EMT (14) through AKT and Gsk $\beta$ (15). ILK signaling activates the key transcription factor SLUG, which upregulates the expression of fibronectin as well as vimentin involved in the EMT process (16). In our PSC sample cohort, higher 

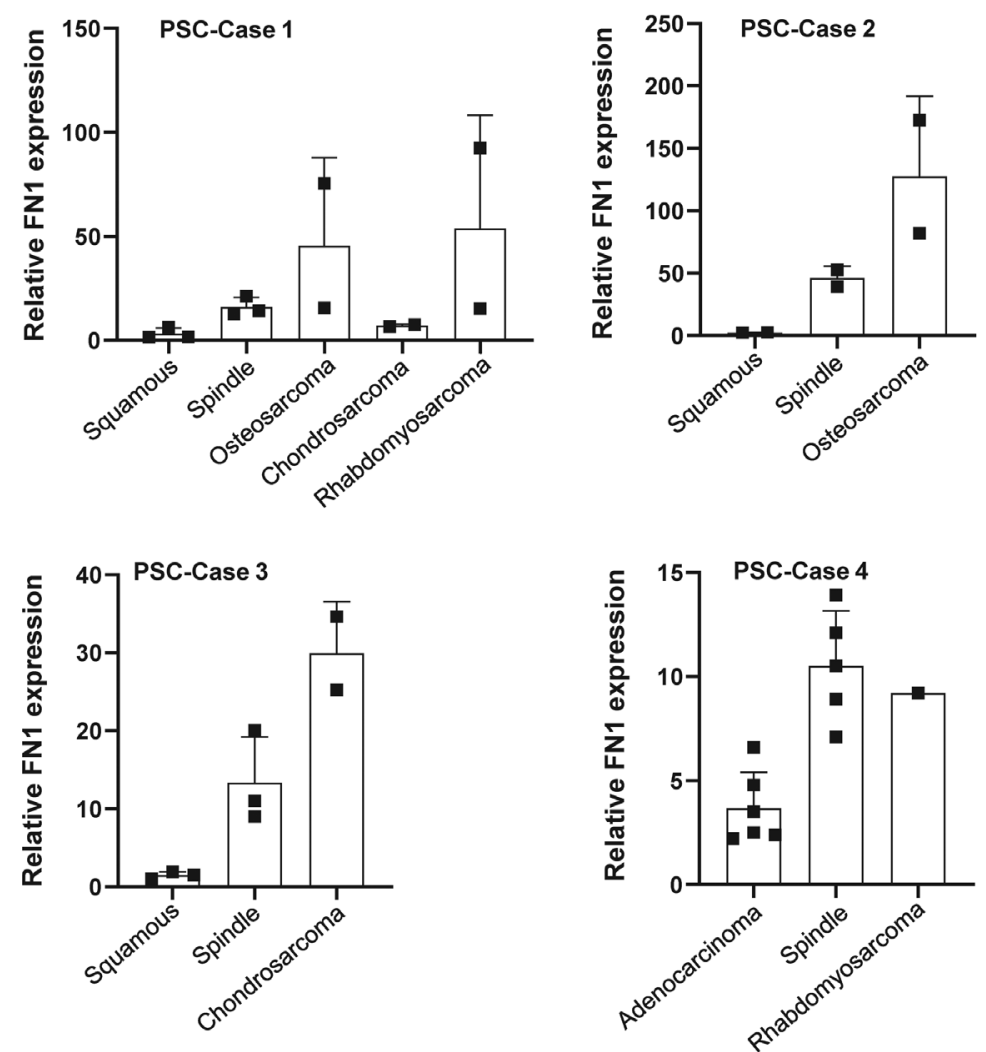

Figure 5. Intratumor expression of fibronectin in the PSCs and epithelial (squamous cell carcinoma and adenocarcinoma) components of the carcinosarcomas (PSC-Cases 1-4). The expression levels of fibronectin were determined by digital spatial profiling. The data represent the mean \pm SD. The expression levels of fibronectin were higher in the sarcoma components than in the epithelial components. Highest expression levels of fibronectin were observed in the osteosarcoma components in PSC cases. PSC, pulmonary sarcomatoid carcinomas.

expression levels of fibronectin were observed at both the transcript and protein levels. Fibronectin has been shown to promote cell motility, opsonization, and cell adhesion in carcinomas $(19,20)$ and to promote EMT $(21)$.

EMT causes resistance to various types of treatments, such as cytotoxic chemotherapy and tyrosine kinase inhibitor therapy (22-24). The hypothesis that PSCs are caused by fibronectin-mediated EMT of carcinomas is consistent with the refractoriness of PSCs to treatment. Our findings allow us to speculate on the involvement of EMT in the progression to sarcoma. In a study of biphasic sarcomatoid carcinomas of the lung, Manzotti et al found that PSC could originate from EMT based on morphological characterization (25). In our study, a more detailed analysis of the EMT status of PSC was yielded through transcriptomic and digital spatial profiling, although focusing on pleomorphic carcinoma of lung. The clinical relevance and possible applications based on our findings can fall into several areas. From the diagnosis and prediction of prognosis perspective, immunostaining of EMT related molecules including fibronectin will be meaningful for understanding the grade of malignancy and possibly predicting of prognosis of PSC patients, although the association of fibronectin expression and prognosis of PSC patients remains unknown it will be meaningful to explore in future studies. From a therapeutic perspective, EMT-targeted therapy is expected to inhibit tumor growth, malignant and resistant transformation. For other cancer types, Fresolimumab (26) targeting N-cadherin, ADH-1, TGF $\beta$, ZEB1, SNAIL2, TWIST, vimentin-targeted metformin (27),
anti-EpCAM immunotoxin targeting EpCAM (28), catumaxomab (29) are possible and other potential candidates are being investigated in clinical trials.

We have also shown increased fibronectin expression in the sarcomatoid components. Thus, targeting fibronectin is one possible treatment approach. Most antibody approaches targeting fibronectin utilized antibodies against the extra-domain B (EDB) domain. A human EDB domain specific antibody (L19) was isolated by Carnemolla et al (30). This antibody has been used for targeted delivery of IL2 as a fused L19-IL2 protein and has demonstrated efficacy in the mouse F9 teratocarcinoma as well as other preclinical tumor models (30). The strategy of targeting fibronectin EDB at tumor sites has been extended with a fusion protein of the L19 fragment with interleukin-12 (IL12) (31), a potent mediator of innate and cell immunity with anti-tumor and anti-metastatic properties $(32,33)$. Other approaches with the L19 antibody have analyzed the use of conjugated photosensitizers and liposomes. Fabbrini et al conjugated L19 to a photosensitizer [bis(triethanolamine)Sn(IV) chlorin e6] that generates toxic oxygen species after irradiation with red light (34). Thus, antibody-based approaches to target fibronectin in the tumor and tumor vasculature and specifically deliver anti-tumor agents to tumors are promising avenues in cancer therapy including the management of PSC.

We also postulated that the ILK signaling pathway may be implicated in the induction of EMT. Therefore, targeting ILK may offer an additional novel therapeutic potential for managing PSCs. Presently, ILK-targeted therapies are being 
investigated in solid tumors. Preclinical studies have shown that knockdown of ILK expression in cancer cells using siRNA or shRNA significantly inactivates the PI3K/AKT pathway and suppresses EMT, tumor growth and metastasis in tongue and prostate cancer cells in vitro and in vivo $(35,36)$. As an ILK inhibitor, Lee et al found that N-methyl-3-(1-(4-(piperazin-1-yl) phenyl)-5-(40-(trifluoromethyl)-[1,10-biphenyl]-4-yl)-1H-pyraz ole-3-yl) propanamide (compound 22) could potently inhibit the growth of prostate and breast cancer cells via the inactivation of the AKT pathway and inhibition of the transcription factor Y-box binding protein-1 (YB-1). Compound 22 was used as a single agent to inhibit the growth of prostate tumor xenografts in vivo (37). In addition, QLT0267, a novel ILK inhibitor, has been reported to reduce tumor volume in thyroid cancer and glioblastoma xenografts $(38,39)$. It was also shown that QLT0267 may inhibit EMT, which is involved in 5-Fluorouracil resistance in colorectal cancer (40). These findings suggest that ILK is an effective therapeutic target for cancer treatment.

We previously showed that the tubulin binder eribulin suppresses TGF $\beta$ - or 5-FU-induced EMT and have the potential to induce mesenchymal epithelial transition (MET) in triple-negative breast cancer cells $(24,41,42)$. Therefore, control of EMT or induction of mesenchymal-epithelial transition are among the treatment strategies for conquering PSCs.

One of the major limitations of this study is the small number of PSC cases, which was unavoidable given the rare nature of PSCs and the difficulty in obtaining surgically resected tissue samples. Here, we focused on carcinosarcoma and pleomorphic carcinoma as the main objective and used adenocarcinoma and squamous carcinoma as a reference. Therefore, a confirmation study using an additional sample cohort that includes pure pulmonary blastoma, spindle cell carcinoma and giant cell carcinoma will be needed to confirm the results of the present study. Unfortunately, we were also unable to microscopically dissect intratumor components for mutation and transcriptome analysis because the samples were unsuitable.

In this study, we demonstrated that digital IHC and NGS provided the molecular profiles of the FFPE specimens of PSCs at the DNA, transcript and protein levels in this study. In particular, DSP enabled comparison of different components in the same tumor tissue and analysis of the intratumor heterogeneity. We consider the results as being consistent with the hypothesis that the ILK-fibronectin mediated EMT process is involved in the pathogenesis of PSCs. Fibronectin may serve as a marker of PSCs.

\section{Acknowledgements}

The authors would like to thank Ms. Keiko Obata (Department of Thoracic Surgery, Kindai University Faculty of Medicine), Mr. Yoshihiro Mine (Center for Instrumental Analyses Central Research Facilities, Kindai University Faculty of Medicine), and Ms. Ayaka Kitano (Department of Genome Biology, Kindai University Faculty of Medicine) for technical assistance provided during the study.

\section{Funding}

The current study was supported in part by a Grant-in Aid for Scientific Research (C) from the Japan Society for the
Promotion of Science (grant no. JP19K07722) and in part by a Grant-in Aid for Scientific Research on Innovative Areas 'Frontier Research on Chemical Communications' (grant nos. JP17H06400 and JP17H06404).

\section{Availability of data and materials}

Data analyzed and material used during the current study are available from the corresponding author on reasonable request.

\section{Authors' contributions}

SS, KS and KN conceived and designed the study. SS, KS, TC, and NS performed the experiments. TM and KN confirmed the authenticity of raw data. SS, KS, TS, and TM performed the statistical analysis. SS, KS, NS, TS, TM, and KN contributed to the analysis and interpretation of data. SS, KS, and $\mathrm{KN}$ wrote the paper. All authors read and approved the final manuscript.

\section{Ethics approval and consent to participate}

The current study was approved by the Institutional Review Board of Kindai University Faculty of Medicine, and written informed consent was obtained from all patients for study participation.

\section{Patient consent for publication}

Written informed consent was obtained from all patients for publication.

\section{Competing interests}

The authors declare that they have no competing interests.

\section{References}

1. Pelosi G, Sonzogni A, De Pas T, Galetta D, Veronesi G, Spaggiari L, Manzotti M, Fumagalli C, Bresaola E, Nappi O, et al: Review article: Pulmonary sarcomatoid carcinomas: A practical overview. Int J Surg Pathol 18: 103-120, 2010.

2. Schrock AB, Li SD, Frampton GM, Suh J, Braun E, Mehra R, Buck SC, Bufill JA, Peled N, Karim NA, et al: Pulmonary sarcomatoid carcinomas commonly harbor either potentially targetable genomic alterations or high tumor mutational burden as observed by comprehensive genomic profiling. J Thorac Oncol 12: 932-942, 2017.

3. Yendamuri S, Caty L, Pine M, Adem S, Bogner P, Miller A, Demmy TL, Groman A and Reid M: Outcomes of sarcomatoid carcinoma of the lung: A surveillance, epidemiology, and end results database analysis. Surgery 152: 397-402, 2012.

4. Travis WD and World Health Organization: International Agency for Research on Cancer, International Association for the Study of Lung Cancer. And International Academy of Pathology: Pathology and genetics of tumours of the lung, pleura, thymus and heart. IARC Press, Oxford University Press (distributor), Lyon, Oxford, 2004.

5. Terra SB, Jang JS, Bi L, Kipp BR, Jen J, Yi ES and Boland JM: Molecular characterization of pulmonary sarcomatoid carcinoma: Analysis of 33 cases. Mod Pathol 29: 824-831, 2016.

6. Weissferdt A: Pulmonary sarcomatoid carcinomas: A review. Adv Anat Pathol 25: 304-313, 2018.

7. Lucas DR, Pass HI, Madan SK, Adsay NV, Wali A, Tabaczka P and Lonardo F: Sarcomatoid mesothelioma and its histological mimics: A comparative immunohistochemical study. Histopathology 42: 270-279, 2003. 
8. Toki MI, Merritt CR, Wong PF, Smithy JW, Kluger HM, Syrigos KN, Ong GT, Warren SE, Beechem JM and Rimm DL: High-plex predictive marker discovery for melanoma immunotherapy-treated patients using digital spatial profiling. Clin Cancer Res 25: 5503-5512, 2019.

9. Vieira T, Antoine M, Ruppert AM, Fallet V, Duruisseaux M, Giroux Leprieur E, Poulot V, Rabbe N, Sclick L, Beau-Faller M, et al: Blood vessel invasion is a major feature and a factor of poor prognosis in sarcomatoid carcinoma of the lung. Lung Cancer 85: 276-281, 2014.

10. Decalf J, Albert ML and Ziai J: New tools for pathology: A user's review of a highly multiplexed method for in situ analysis of protein and RNA expression in tissue. J Pathol 247: 650-661, 2019.

11. Alborelli I, Leonards K, Rothschild SI, Leuenberger LP, Savic Prince S, Mertz KD, Poechtrager S, Buess M, Zippelius A, Läubli $\mathrm{H}$, et al: Tumor mutational burden assessed by targeted NGS predicts clinical benefit from immune checkpoint inhibitors in non-small cell lung cancer. J Pathol 250: 19-29, 2020.

12. Cao D, Xu H, Xu X, Guo T and Ge W: High tumor mutation burden predicts better efficacy of immunotherapy: A pooled analysis of 103078 cancer patients. Oncoimmunology 8: e1629258, 2019.

13. Heeke S, Benzaquen J, Long-Mira E, Audelan B, Lespinet V, Bordone O, Lalvée S, Zahaf K, Poudenx M, Humbert O, et al: In-house implementation of tumor mutational burden testing to predict durable clinical benefit in non-small cell lung cancer and melanoma patients. Cancers (Basel) 11: 1271, 2019.

14. Yilmaz M and Christofori G: EMT, the cytoskeleton, and cancer cell invasion. Cancer Metastasis Rev 28: 15-33, 2009.

15. Hannigan G, Troussard AA and Dedhar S: Integrin-linked kinase: A cancer therapeutic target unique among its ILK. Nat Rev Cancer 5: 51-63, 2005.

16. Fenouille N, Tichet M, Dufies M, Pottier A, Mogha A, Soo JK, Rocchi S, Mallavialle A, Galibert MD, Khammari A, et al: The epithelial-mesenchymal transition (EMT) regulatory factor SLUG (SNAI2) is a downstream target of SPARC and AKT in promoting melanoma cell invasion. PLoS One 7: e40378, 2012.

17. Liang X, Li Q, Xu B, Hu S, Wang Q, Li Y, Zong Y, Zhang S and Li C: Mutation landscape and tumor mutation burden analysis of Chinese patients with pulmonary sarcomatoid carcinomas. Int J Clin Oncol 24: 1061-1068, 2019.

18. Velcheti V, Rimm DL and Schalper KA: Sarcomatoid lung carcinomas show high levels of programmed death ligand-1 (PD-L1) J Thorac Oncol 8: 803-805, 2013.

19. Gopal S, Veracini L, Grall D, Butori C, Schaub S, Audebert S, Camoin L, Baudelet E, Radwanska A, Beghelli-de la Forest Divonne S, et al: Fibronectin-guided migration of carcinoma collectives. Nat Commun 8: 14105, 2017.

20. Wang WY, Twu CW, Liu YC, Lin HH, Chen CJ and Lin JC: Fibronectin promotes nasopharyngeal cancer cell motility and proliferation. Biomed Pharmacother 109: 1772-1784, 2019.

21. Rick JW, Chandra A, Dalle Ore C, Nguyen AT, Yagnik G and Aghi MK: Fibronectin in malignancy: Cancer-specific alterations, protumoral effects, and therapeutic implications. Semin Oncol 46: 284-290, 2019

22. Park SY, Kim MJ, Park SA, Kim JS, Min KN, Kim DK, Lim W, Nam JS and Sheen YY: Combinatorial TGF- $\beta$ attenuation with paclitaxel inhibits the epithelial-to-mesenchymal transition and breast cancer stem-like cells. Oncotarget 6: 37526-37543, 2015.

23. Sarkar FH, Li Y, Wang Z and Kong D: Pancreatic cancer stem cells and EMT in drug resistance and metastasis. Minerva Chir 64: 489-500, 2009.

24. Terashima M, Sakai K, Togashi Y, Hayashi H, De Velasco MA, Tsurutani J and Nishio K: Synergistic antitumor effects of S-1 with eribulin in vitro and in vivo for triple-negative breast cancer cell lines. Springerplus 3: 417, 2014

25. Manzotti G, Torricelli F, Benedetta D, Lococo F, Sancisi V, Rossi G,Piana S and Ciarrocchi A: An Epithelial-to-mesenchymal transcriptional switch triggers evolution of pulmonary sarcomatoid carcinoma (PSC) and identifies dasatinib as new therapeutic option. Clin Cancer Res 25: 2348-2360, 2019.
26. Singh M, Yelle N, Venugopal C and Singh SK: EMT: Mechanisms and therapeutic implications. Pharmacol Ther 182: 80-94, 2018.

27. Kordes S, Pollak MN, Zwinderman AH, Mathôt RA, Weterman MJ, Beeker A, Punt CJ, Richel DJ and Wilmink JW: Metformin in patients with advanced pancreatic cancer: A double-blind, randomised, placebo-controlled phase 2 trial. Lancet Oncol 16: 839-847, 2015.

28. Andersson Y, Inderberg EM, Kvalheim G, Herud TM, Engebraaten O, Flatmark K, Dueland S and Fodstad Ø: Immune stimulatory effect of anti-EpCAM immunotoxin-improved overall survival of metastatic colorectal cancer patients. Acta Oncol 59: 404-409, 2020

29. Mau-Sørensen M, Dittrich C, Dienstmann R, Lassen U, Büchler W, Martinius H and Tabernero J: A phase I trial of intravenous catumaxomab: A bispecific monoclonal antibody targeting EpCAM and the T cell coreceptor CD3. Cancer Chemother Pharmacol 75: 1065-1073, 2015.

30. Carnemolla B, Neri D, Castellani P, Leprini A, Neri G, Pini A, Winter $G$ and Zardi L: Phage antibodies with pan-species recognition of the oncofoetal angiogenesis marker fibronectin ED-B domain. Int J Cancer 68: 397-405, 1996.

31. Halin C, Rondini S, Nilsson F, Berndt A, Kosmehl H, Zardi L and Neri D: Enhancement of the antitumor activity of interleukin-12 by targeted delivery to neovasculature. Nat Biotechnol 20: 264-269, 2002

32. Tsung K, Meko JB, Peplinski GR, Tsung YL and Norton JA: IL-12 induces T helper 1-directed antitumor response. J Immunol 158: 3359-3365, 1997.

33. Brunda MJ, Luistro L, Warrier RR, Wright RB, Hubbard BR, Murphy M, Wolf SF and Gately MK: Antitumor and antimetastatic activity of interleukin 12 against murine tumors. J Exp Med 178: 1223-1230, 1993.

34. Fabbrini M, Trachsel E, Soldani P, Bindi S, Alessi P, Bracci L, Kosmehl H, Zardi L, Neri D and Neri P: Selective occlusion of tumor blood vessels by targeted delivery of an antibody-photosensitizer conjugate. Int J Cancer 118: 1805-1813, 2006.

35. Xing Y, Qi J, Deng S, Wang C, Zhang L and Chen J: Small interfering RNA targeting ILK inhibits metastasis in human tongue cancer cells through repression of epithelial-to-mesenchymal transition. Exp Cell Res 319: 2058-2072, 2013.

36. Yuan Y, Xiao Y, Li Q, Liu Z, Zhang X, Qin C, Xie J, Wang X and $\mathrm{Xu} \mathrm{T}$ : In vitro and in vivo effects of short hairpin RNA targeting integrin-linked kinase in prostate cancer cells. Mol Med Rep 8: 419-424, 2013

37. Lee SL, Hsu EC, Chou CC, Chuang HC, Bai LY, Kulp SK and Chen CS: Identification and characterization of a novel integrin-linked kinase inhibitor. J Med Chem 54: 6364-6374, 2011.

38. Edwards LA, Woo J, Huxham LA, Verreault M, Dragowska WH, Chiu G, Rajput A, Kyle AH, Kalra J, Yapp D, et al: Suppression of VEGF secretion and changes in glioblastoma multiforme microenvironment by inhibition of integrin-linked kinase (ILK). Mol Cancer Ther 7: 59-70, 2008.

39. Younes MN, Kim S, Yigitbasi OG, Mandal M, Jasser SA Dakak Yazici Y, Schiff BA, El-Naggar A, Bekele BN, Mills GB and Myers JN: Integrin-linked kinase is a potential therapeutic target for anaplastic thyroid cancer. Mol Cancer Ther 4: 1146-1156, 2005.

40. Tsoumas D, Nikou S, Giannopoulou E, Champeris Tsaniras S, Sirinian C, Maroulis I, Taraviras S, Zolota V, Kalofonos HP and Bravou V: ILK expression in colorectal cancer is associated with EMT, cancer stem cell markers and chemoresistance. Cancer Genomics Proteomics 15: 127-141, 2018.

41. Iwasa T, Tsurutani J, Watanabe S, Kato R, Mizuno Y, Kojima Y, Takashima T, Matsunami N, Morimoto T, Yamamura J, et al: Multicentre, phase II study of eribulin in combination with S-1 in patients with advanced breast cancer. BMC Cancer 19: 962, 2019.

42. Sakiyama T, Tsurutani J, Iwasa T, Kawakami H, Nonagase Y, Yoshida T, Tanaka K, Fujisaka Y, Kurata T, Komoike Y, et al: A phase I dose-escalation study of eribulin and S-1 for metastatic breast cancer. Br J Cancer 112: 819-824, 2015. 